\title{
In vitro dynamic pharmacokinetic/ pharmacodynamic(PK/PD) modeling and PK/PD cutoff of cefquinome against Haemophilus parasuis
}

Xia Xiao ${ }^{1+}$, Jian Sun ${ }^{1+}$, Yi Chen ${ }^{1}$, Rui-Juan Huang ${ }^{1}$, Ting Huang ${ }^{1}$, Guilin Gary Qiao ${ }^{2}$, Yu-Feng Zhou ${ }^{1}$ and Ya-Hong Liu, ${ }^{1, *}$

\begin{abstract}
Background: Haemophilus parasuis (H. parasuis) causes Glässer's disease and multisystem infectious disease. It is one of the major causes of nursery mortality in swine herds. Cefquinome (CEQ) is proposed for the treatment of pigs against respiratory tract infection. However, few studies have investigated the PK/PD characteristics and PK/PD cutoff of this drug against $H$. parasuis.

Results: A total of $213 \mathrm{H}$. parasuis strains were isolated from diseased pigs in China. The minimal inhibitory concentrations (MICS) of CEQ against these isolates were determined. The $\mathrm{MIC}_{50}$ and $\mathrm{MIC}_{90}$ values were 0.125 and $8 \mathrm{mg} / \mathrm{L}$, respectively. An in vitro dynamic PK/PD infection model was used to investigate the antimicrobial effect of CEQ against $H$. parasuis strain of serotype 5 . The target values of CEQ for $3-\log _{10}$-unit and $4-\log _{10}$-unit decreases effects were the percent time that CEQ concentrations were above the minimum inhibitory concentration (T\% > MIC) of 61 and 71 respectively. According to Monte Carlo simulation, the PK/PD cutoff for CEQ against $H$. parasuis was $0.06 \mathrm{mg} / \mathrm{L}$. The suggested dose regimen was $4 \mathrm{mg} / \mathrm{kg} / 12 \mathrm{~h} \mathrm{BW}$.

Conclusions: The value of PK/PD surrogate marker T\% > MIC is of great utility in CEQ clinical usage. The very first CEQ PK/PD cutoff provide fundamental data for CEQ breakpoint determination. A more desirable dose regimen against $H$. parasuis was provided for CEQ using in China district.
\end{abstract}

Keywords: Pharmacokinetic/pharmacodynamic, Cefquinome, PK/PD cutoff, Monte Carlo simulation

\section{Background}

$H$. parasuis is a commensal bacterium of the upper respiratory tract of swine [1]. Under certain conditions, $H$. parasuis can invade the body and cause Glässer's disease as well as other severe diseases, such as arthritis, fibrinous polyserositis and meningitis [2]. Moreover, H. parasuis can invade into the AOC- 45 porcine aorta endothelial cells [3] and its endotoxin is able to induce

\footnotetext{
* Correspondence: gale@scau.edu.cn

${ }^{\dagger}$ Equal contributors

${ }^{1}$ College of Veterinary Medicine, National Reference Laboratory of Veterinary Drug Residues (SCAU), South China Agricultural University, Guangzhou 510642, China

${ }^{3}$ Jiangsu Co-Innovation Centre for Prevention and Control of Important Animal Infectious Diseases and Zoonoses, Yangzhou, Jiangsu,

People's Republic of China

Full list of author information is available at the end of the article
}

disseminated intravascular coagulation, which results in the formation of microthrombi in some important organs [2]. In addition, $H$. parasuis frequently interacts with porcine reproductive respiratory syndrome virus (PRRSv) and causes more economic losses [4,5]. $H$. parasuis has numerous serovars, some of which show severe virulence and cause death within 4 days such as serovar 1, 5, 10,12, 13 and 14 [6]. Of them, serovar 5 and 4 are the most prevalent types isolated in China [7], Danmark [8], Germany [6], the USA [9], Japan [10] and Spain [11], whereas serovars 5 and 13 are prevalent in Australia [12]. Due to the serovar diversity and lack of cross-reaction among the serovars, it is hard to develop an effective cross-protective vaccine [13]. Therefore 
pharmacotherapy still plays a significant role in treating H. parasuis infections.

Cefquinome (CEQ) is a fourth generation cephalosporin antibiotic that is solely developed for veterinary use. It possesses high stability to $\beta$-lactamases and is active against Gram-positive and Gram-negative bacteria. The European Medicines Agency (EMA) proposes CEQ for the treatment of pigs against respiratory tract infections with a dosage regimen of $2 \mathrm{mg} / \mathrm{kg} / 24 \mathrm{~h}$ bodyweight (BW) for three to five days [14]. As one of the major pathogens of respiratory tract of pigs, few studies have investigated the killing pattern and PK/PD characteristics of this drug against $H$. parasuis, moreover, the Clinical and Laboratory Standards Institute (CLSI) subcommittee on Veterinary Antimicrobial Susceptibility Testing (VAST) which establishes veterinary clinically breakpoints [15] has not established breakpoints for $H$. parasuis. The value of PK/PD surrogate which is of great utility in CEQ clinical usage and the CEQ PK/PD cutoff providing fundamental data for CEQ breakpoint determination should be illuminated.

In this study, the minimal inhibitory concentrations (MICs) of CEQ against $213 \mathrm{H}$. parasuis isolates identified in China were determined. An in vitro $\mathrm{PK} / \mathrm{PD}$ infection model has been used to investigate the antimicrobial effect of CEQ against $H$. parasuis strain of serotype 5 , which is highly virulent and one of the most prevalent serotypes in China [7]. A 5,000-subjects Monte Carlo simulation has been done to derive a PK/PD cutoff based on three aspects: MIC distributions of CEQ against $H$. parasuis, pharmacokinetic/pharmacodynamic (PK/PD) indices and Pharmacokinetics of CEQ in swine obtained in a previous study [16]. A more rational regimen was recommended according to Monte Carlo simulation results.

\section{Methods}

Animal ethics

All husbandry practices and experimental operations were performed with full consideration of animal welfare. Research ethical approval was granted by the South China Agriculture University Animal ethics committee (2014-03).

\section{Strains and antibiotic}

H. parasuis strain representing serovar 5 was kindly provided by Professor Ming Liao, College of Veterinary Medicine, South China Agricultural University, Guangzhou, Guangdong Province, China. It was CEQ sensitive to MIC of $0.003 \mathrm{mg} / \mathrm{L}$. The other $213 \mathrm{H}$. parasuis isolates used in this study were derived from diseased pigs suffering fibrinous polyserositis, meningitis and polyarthritis diagnosed in China during August 2010 to July 2011. Serotypes of these isolates are not known. All the strains were stored at $-80^{\circ} \mathrm{C}$ in milk. Prior to use, they were streaked on a tryptone soya agar (TSA) (Oxoid Ltd., Basingstoke, Hampshire, UK) added with 5\% new-born calf serum (Guangzhou Ruite Bio-tec Co., Ltd., Guangdong, China) and $2 \%$ beta-Nicotinamide adenine dinucleotide trihydrate (NAD) (Qingdao Hope Bio-Technology Co., Ltd,, Shandong, China). Tryptone soya broth (Oxoid Ltd., Basingstoke, Hampshire, UK) added with the same amount of NAD and new-born calf serum was used to culture $H$. parasuis. CEQ sulfate injection $(25 \mathrm{mg} / \mathrm{mL})$ was purchased from Hebei Yuanzheng Pharmaceutical Company (Hebei, China).

\section{MICs}

Given the unavailability of a CLSI approval method for $H$. parasuis, MICs were conducted in accordance with the CLSI VET01-A4 recommendations for Actinobacillus pleuropneumoniae [17]. The quality control strain was Actinobacillus pleuropneumoniae ATCC 27090. The values of $\mathrm{MIC}_{50}$ and $\mathrm{MIC}_{90}$, inhibiting the growth of at least $50 \%$ and $90 \%$ of isolates in a test population, respectively, were calculated in this study following the methods previously published [18].

\section{In vitro dynamic PK/PD modeling}

The in vitro one-compartment PK/PD infection model was constructed according to previously described method [19] with some improvements. The model system contained fresh trypticase soy broth reservoir, central compartment and waste storage compartment. These compartments were connected with silicone tubes. The broth was pumped from the reservoir to the central compartment through a peristaltic pump, which is controlled digitally. An inverted $15-\mathrm{mL}$ centrifuge tube with a cellulose ester membrane $(0.2-\mu \mathrm{m}$ pore size $)$ covering the top was placed in the central compartment to prevent bacteria from flowing out to the medium. Below the membrane, a magnetic stir bar was placed on the bottom of the central compartment. The stir bar mixed the broth and enabled the drug to fully contact with the bacteria. The temperature of the reservoir and central compartment was controlled between 36 and $37^{\circ} \mathrm{C}$ via water bath. The entire experimental system was placed in a UV-sterilized worktable. The reservoir and central compartment contained 700 and $60 \mathrm{~mL}$ of trypticase soy broth, respectively. Fresh broth pumped into the central compartment, and the same volume culture media pumped into the waste container. To simulate intravenous injection pharamcokinetics of CEQ in swine with this equipment, the flow rate was $0.37 \mathrm{~mL} / \mathrm{min}$. The drug was administered into the central compartment via the sampling port at zero time point. 


\section{In vitro time kill curves of CEQ}

A $12 \mathrm{~h}$ culture of bacteria with optical density (OD) value of approximately 0.09 was added to the central compartment $\left[10^{7}\right.$ colony forming unit $\left.(\mathrm{cfu}) / \mathrm{mL}\right]$ and incubated at $35^{\circ} \mathrm{C}$ to $37^{\circ} \mathrm{C}$ for $30 \mathrm{~min}$ to condition the bacteria to the new environment. Different doses of CEQ $(0.28,0.68,4.03,9.79,15.26,37.08,90.13,140.50$, $219.03,341.45 \mu \mathrm{g}$ ) or control (sterile normal saline) were introduced into the central compartment, and the peristaltic pump was turned on immediately. A $150 \mu \mathrm{L}$ aliquot was obtained for bacterial counting at time points of 0, 3, 6, 9, 12 and $24 \mathrm{~h}$. Samples were diluted properly with sterile normal saline, and $25-\mu \mathrm{L}$ of aliquots of the last four diluted samples were plated onto the TSA plates and incubated at $37^{\circ} \mathrm{C}$ for $24 \mathrm{~h}$. To monitor drug concentrations, $200-\mu \mathrm{L}$ aliquots were also obtained at $1,6,12$ and $24 \mathrm{~h}$ and centrifuged at $8,000 \mathrm{rpm}$ at $4^{\circ} \mathrm{C}$ for $10 \mathrm{~min}$. The supernatant was stored at $-80^{\circ} \mathrm{C}$ and analyzed within 1 month. All experiments were performed in duplicate on different days.

\section{Pharmacokinetics and PK/PD analysis}

The samples were analyzed for CEQ concentration using the method reported previously [19]. The PK data were analyzed using Phoenix WinNonlin 6.0 software (Pharsight Co. Ltd.). A T\% > MIC value during a 24-h interval was calculated using the pharmacokinetic and MIC data for each time kill curve. The in vitro drug effect was quantified by changes in $\log _{10}$ cfu counts between 24 and $0 \mathrm{~h}$. Data were analyzed using sigmoid $E_{\max }$ model WINNONLIN software (version 6.1; Pharsight, CA, USA) per the following equation:

$$
E=E_{0}+\frac{E_{\max } \times C_{e}^{N}}{E C_{50}^{N}+C_{e}^{N}}
$$

where $E_{0}$ is the change in $\log _{10} \mathrm{cfu} / \mathrm{mL}$ after 24-h incubation in the control sample compared to the initial inoculum. $E_{\max }$ is the difference in effect between the greatest amount of growth (as seen for the growth control, $\mathrm{E}_{0}$ ) and the greatest amount of kill. $C_{\mathrm{e}}$ is the T\% > MIC in the effect compartment. $\mathrm{EC}_{50}$ is the $\mathrm{T} \%>\mathrm{MIC}$ value producing a $50 \%$ reduction in bacterial counts from the initial inoculum, and $N$ is the Hill coefficient that describes the steepness of the T\%>MIC-effect curve. Three levels of growth inhibition were calculated. $\mathrm{T} \%>$ MIC for bacteriostatic and bactericidal actions are values that produce $E=0$ (no change in bacterial counts after $24 \mathrm{~h}$ incubation) and $E=-3$ (a $3 \log 10$ or $99.9 \%$ reduction of the original inoculum counts after $24 \mathrm{~h}$ incubation), respectively. Bacterial eradication is the lowest $\mathrm{T} \%>$ MIC that provides a $4 \log _{10}$ reduction.

\section{Monte Carlo simulation}

Based on a previous pharmacokinetic study of CEQ in pigs [19], MIC distribution, and the value of $\mathrm{PK} / \mathrm{PD}$ target indices obtained in this study, a 5,000-subjects Monte Carlo simulation was conducted using Crystal Ball Professional V7.2.2 software. The time above MIC was calculated using the following equation:

$$
C=\frac{k_{a} F X_{0}}{k_{a}-k}\left(e^{-k t}-e^{-K_{a} t}\right)
$$

Where $C$ is the value of MIC, $k_{a}$ is the absorption half life, $\mathrm{F}$ is the bioavailability, $\mathrm{X}_{0}$ is the dose of antibiotic and $\mathrm{k}$ is the elimination half life.

All the PK parameters were assumed to be normally distributed in the form of mean values and confidence intervals (Table 1). MICs were fixed at single values from $0.0015 \mathrm{mg} / \mathrm{L}$ to $16 \mathrm{mg} / \mathrm{L}$. The PK/PD cutoff is the MIC, at which the probability of target attainment (PTA) for $3-\log _{10}$-unit decrease equals $90 \%$ under clinical recommended dose. Scenarios were simulated separately for the IM administration, which were single-dose administrations of $2,4,8,16,32$ and $60 \mathrm{mg} / \mathrm{kg} \mathrm{BW}$ and doses of $1,2,4,6,8$ and $16 \mathrm{mg} / \mathrm{kg} \mathrm{BW}$ administered in two equal doses at 12 -h interval.

\section{Results}

\section{MIC distribution}

MICs of CEQ against $213 \mathrm{H}$. parasuis isolates were diverse, ranging from $0.0015 \mathrm{mg} / \mathrm{L}$ to $16 \mathrm{mg} / \mathrm{L}$. The percentages for each MIC $(0.0015,0.003,0.006,0.015,0.03$, $0.06,0.125,0.25,0.5,1,2,4,8$ and $16 \mathrm{mg} / \mathrm{L})$ were $1.48 \%$, $0.96 \%, 2.95 \%, 3.94 \%, 13.79 \%, 16.75 \%, 17.73 \%, 19.21 \%$, $2.96 \%, 5.42 \%, 0.99 \%, 2.46 \%, 7.39 \%$ and $3.94 \%$, respectively (Figure 1). Although a low peak of MIC was observed at $8 \mathrm{mg} / \mathrm{L}$, the MICs distributed in a normal distribution pattern basically with a definite peak at 0.25 $\mathrm{mg} / \mathrm{L}$. The $\mathrm{MIC}_{50}$ and $\mathrm{MIC}_{90}$ were determined to be 0.125 and $8 \mathrm{mg} / \mathrm{L}$, respectively.

\section{In vitro dynamic PK/PD modeling}

The pharmacokinetics of CEQ in pigs was well simulated by this in vitro model with a relative deviation below $6 \%$. For bacterial counting, the limit of determination was 400

Table 1 Serum pharmacokinetic parameters after IV administration at $2 \mathrm{mg} / \mathrm{kg}$ bodyweight in a two-compartment open model used for Monte Carlo simulation

\begin{tabular}{lll}
\hline Parameter (units) & Mean value & SD \\
\hline $\mathrm{T}_{1 / 2 \mathrm{ka}}(\mathrm{h})$ & 0.06 & 0.03 \\
$\mathrm{~T}_{1 / 2 \beta}(\mathrm{h})$ & 2.34 & 0.09 \\
$\mathrm{CL} / \mathrm{F}(\mathrm{L} / \mathrm{kg})$ & 0.09 & 0.03 \\
$\mathrm{~F}(\%)$ & 116.29 & 14.72 \\
\hline
\end{tabular}




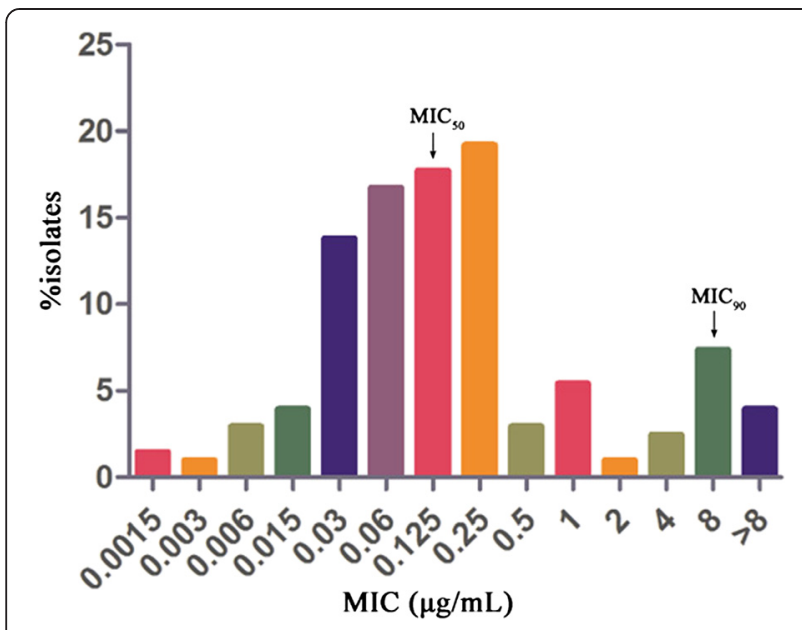

Figure 1 Minimum inhibitory concentrations (MIC) of cefquinome against $\boldsymbol{H}$. parasuis. (213 strains in total).

cfu/mL. The CEQ inhibited $H$. parasuis moderately when $\mathrm{T} \%>\mathrm{MIC}$ values were equal to or below 15 within $24 \mathrm{~h}$ (Figure 2). When T\% > MIC value were 35 and 50, CEQ gained a $3-\log _{10}$-unit and a $4-\log _{10}$-unit decrease, respectively, at $9 \mathrm{~h}$ to $12 \mathrm{~h}$, but regrowth was observed at $24 \mathrm{~h}$. When T\% > MIC increased to the values of 60 and 70 , CEQ completely killed $H$. parasuis without regrowth in $24 \mathrm{~h}$ (3-log-unit and 4-log-unit decrease, respectively). CEQ is considered to be a time-dependent drug. The in vitro antimicrobial effect of CEQ was described successfully using sigmoid $E_{\max }$ model by the suitable PK/PD surrogate marker T\% $>$ MIC (Figure 3). The estimated $E_{0}$, $\mathrm{EC}_{50}$ and $E_{\max }$ are listed in Table 2. The target values of 3- $\log _{10}$-unit and 4- $\log _{10}$-unit decreases for T\% > MIC were 61 and 71 , respectively.

\section{Monte Carlo simulation}

The PTAs following CEQ administration at dose of $2 \mathrm{mg} /$ $\mathrm{kg} / 24 \mathrm{~h}$ was shown in Figure 4. For the recommended

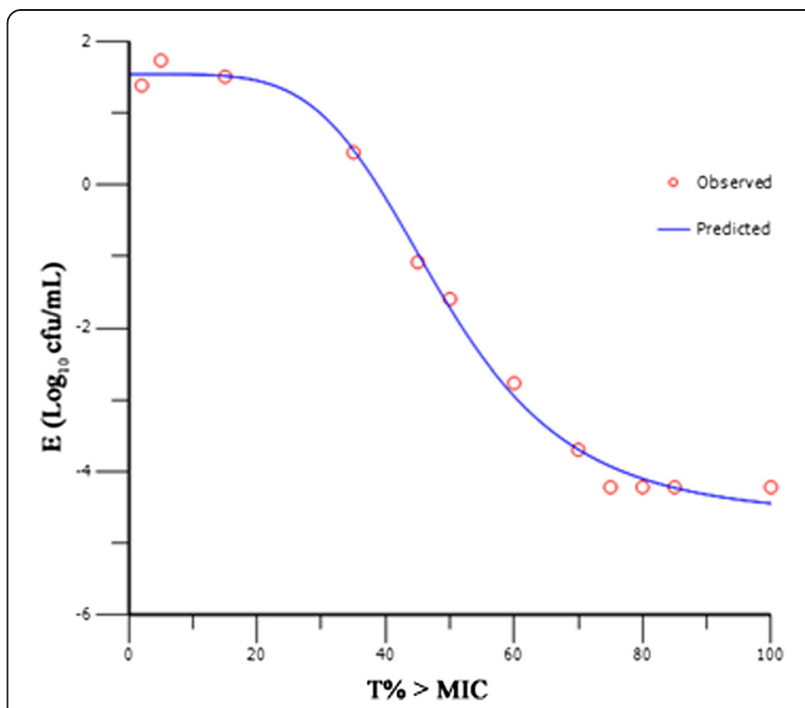

Figure 3 Sigmoid $E_{\max }$ model relationships between antimycoplasmal effect $\left[E, \log _{10}(\mathrm{cfu} / \mathrm{mL})\right]$ and T\% $>$ MIC of cefquinome in in vitro $\mathrm{PK} / \mathrm{PD}$ model against $H$. parasuis with an inoculum size of $1 \times 10^{7} \mathrm{cfu} / \mathrm{mL}$.

$2 \mathrm{mg} / \mathrm{kg} / 24 \mathrm{~h}$ BW dose administered by IM, PTA $>90 \%$ could only be achieved for MIC $<0.06 \mathrm{mg} / \mathrm{L}$. That is to say, the PK/PD cutoff for CEQ against $H$. parasuis was $0.06 \mathrm{mg} / \mathrm{L}$. The PTAs for each effect and the PK/PD cutoffs with different drug regimens are listed in Table 3. A recommended single IM administration of CEQ dose ( $2 \mathrm{mg} / \mathrm{kg} / 24 \mathrm{~h} \mathrm{BW}$ ) could not achieve PTA $>90 \%$, neither did an increased dose of $60 \mathrm{mg} / \mathrm{kg} / 24 \mathrm{~h} \mathrm{BW}$ for all the strains used in this study. However, a PTA of $90.03 \%$ was acquired by splitting the daily dose of $(32 \mathrm{mg} / \mathrm{kg} / 24 \mathrm{~h}$ $\mathrm{BW})$ into two equal doses given at a $12-\mathrm{h}$ interval $(16 \mathrm{mg} /$ $\mathrm{kg} / 12 \mathrm{~h})$. In consideration of the PTA $(85.79 \%)$ following CEQ administration at dose of $4 \mathrm{mg} / \mathrm{kg} / 12 \mathrm{~h}$, which was close to $90 \%$, the recommended dose regimen was defined as $4 \mathrm{mg} / \mathrm{kg} / 12 \mathrm{~h}$. The PTAs following CEQ administration at dose of $4 \mathrm{mg} / \mathrm{kg} / 12 \mathrm{~h}$ was shown in Figure 5 .

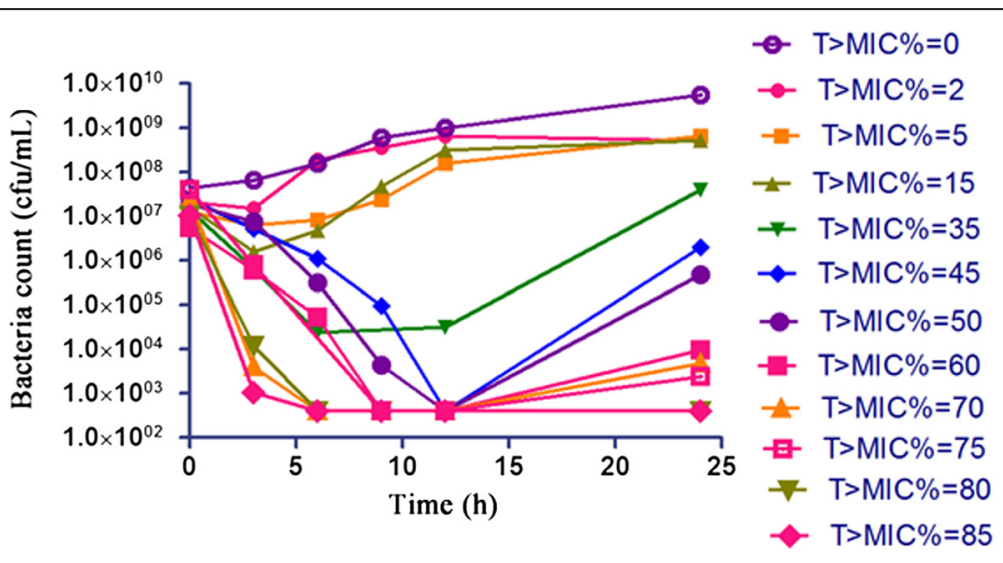

Figure 2 Time-kill curve of cefquinome against $H$. parasuis in in vitro PK/PD model. 
Table 2 Pharmacodynamic analysis of data acquired from in vitro time-killing studies for cefquinome against H. parasuis

\begin{tabular}{ll}
\hline Parameter (units) & Value \\
\hline $\log E_{\max }(\mathrm{cfu} / \mathrm{mL})$ & -4.7 \\
$\log E_{0}(\mathrm{cfu} / \mathrm{mL})$ & 1.6 \\
$\mathrm{EC}_{50}(\mathrm{~h})$ & 48.9 \\
T\% > MIC (bacteriostatic) (h) & 38.7 \\
T\% > MIC (bactericidal) (h) & 60.9 \\
T\% > MIC (bacterial elimination) (h) & 70.9 \\
Slope (N) & 4.7 \\
\hline
\end{tabular}

Where $E_{0}$ is the change in $\log _{10} \mathrm{cfu} / \mathrm{mL}$ after $24 \mathrm{~h}$ incubation in the control sample compared to the initial inoculum. $E_{\max }$ is the difference in effect between the greatest amount of growth (as seen for the growth control, $E_{0}$ ) and the greatest amount of kill. $C_{\mathrm{e}}$ is the T\% $>$ MIC in the effect compartment. $E C_{50}$ is the T\% $>$ MIC value producing a $50 \%$ reduction in bacterial counts from the initial inoculum, and $N$ is the Hill coefficient that describes the steepness of the T\% > MIC-effect curve.

\section{Discussion}

$H$. parasuis frequently causes Glässer's disease, which is often treated with sulfanilamide, quinolones and cephalosporins. However, with these drugs wildly used, the resistant $H$. parasuis isolates are emerging quickly [20]. The most important factor in resistance emergence and spread is drug exposure, especially, the exposure to subtherapeutic drug concentrations [21]. The PK/PD modeling determines the exposure-activity relationships. Using the regimens based on appropriate PK/PD targets will prevent the emergence of resistance [22]. So it is of great importance to administer antibiotics to animals using regimens that will attain appropriate PK/PD targets [23]. In this $\mathrm{PK} / \mathrm{PD}$ analysis, T\% $>$ MIC which was considered as the PK/PD index of most cephalosporins [24] correlated well with in vitro drug efficiency. Through the Sigmoid $E_{\max }$ modeling, the $\mathrm{PK} / \mathrm{PD}$ target for bactericidal effect and elimination action was $\mathrm{T} \%>\mathrm{MIC}=61$ and 71 , respectively. The results reported here were highly consistent with the published data that the greatest efficacy of

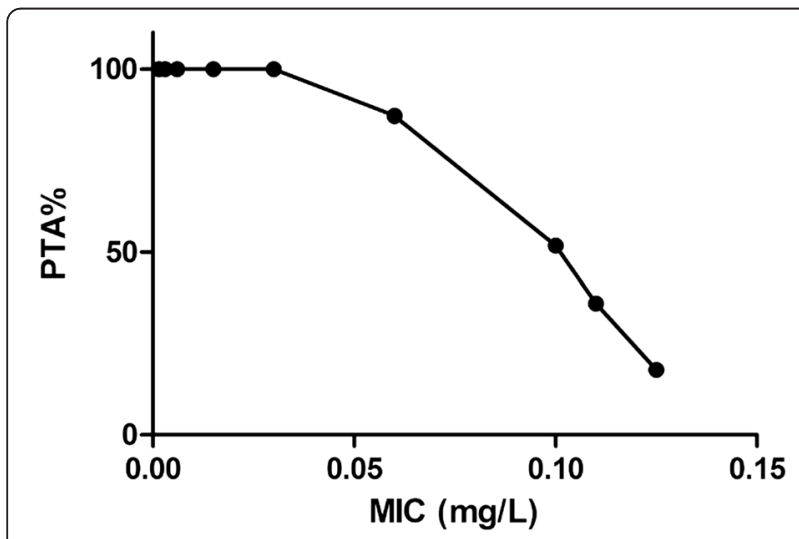

Figure 4 Probability of target attainment (PTA) for the treatment with cefquinome dose of $2 \mathrm{mg} / \mathrm{kg} / 24 \mathrm{~h}$.
Table 3 Probability of target attainment (PTA) and breakpoints of cefquinome against $H$. parasuis with recommended regimens ( $2 \mathrm{mg} / \mathrm{kg} / 24 \mathrm{~h}$ and $4 \mathrm{mg} / \mathrm{kg} / 12 \mathrm{~h}$ )

\begin{tabular}{llll}
\hline Drug regimens & $\begin{array}{l}\text { PTA for } \\
\text { T\% }>\text { MIC }=61\end{array}$ & $\begin{array}{l}\text { PTA for } \\
\text { T\% }>\text { MIC }=71\end{array}$ & $\begin{array}{l}\text { Susceptibility } \\
\text { breakpoint (mg/L) }\end{array}$ \\
\hline $1 \mathrm{mg} / \mathrm{kg} / 12 \mathrm{~h}$ & 76.87 & 72.52 & 0.3 \\
$2 \mathrm{mg} / \mathrm{kg} / 12 \mathrm{~h}$ & 82.19 & 80.63 & 0.6 \\
$4 \mathrm{mg} / \mathrm{kg} / 12 \mathrm{~h}$ & 85.79 & 84.37 & 1.3 \\
$6 \mathrm{mg} / \mathrm{kg} / 12 \mathrm{~h}$ & 86.46 & 85.88 & 1.9 \\
$8 \mathrm{mg} / \mathrm{kg} / 12 \mathrm{~h}$ & 87.64 & 86.76 & 2.6 \\
$16 \mathrm{mg} / \mathrm{kg} / 12 \mathrm{~h}$ & 90.03 & 88.55 & 5.2 \\
$2 \mathrm{mg} / \mathrm{kg} / 24 \mathrm{~h}$ & 44.79 & 28.18 & 0.06 \\
$4 \mathrm{mg} / \mathrm{kg} / 24 \mathrm{~h}$ & 61.77 & 43.52 & 0.15 \\
$8 \mathrm{mg} / \mathrm{kg} / 24 \mathrm{~h}$ & 76.91 & 61.6 & 0.3 \\
$16 \mathrm{mg} / \mathrm{kg} / 24 \mathrm{~h}$ & 81.25 & 77.21 & 0.6 \\
$32 \mathrm{mg} / \mathrm{kg} / 24 \mathrm{~h}$ & 86.06 & 82.21 & 1.2 \\
$60 \mathrm{mg} / \mathrm{kg} / 24 \mathrm{~h}$ & 86.64 & 84.74 & 2.2 \\
\hline
\end{tabular}

cephalosporins in several animal infection models could be achieved as the value of $\mathrm{T} \%>$ MIC reached $60 \%-70 \%$ of the dosing interval against Streptococci or Enterobacteriaceae [25].

PK/PD cutoffs are important tools to set susceptibility breakpoints, which have also been used by regulatory agencies, such as EUCAST and VAST, to refine the susceptibility breakpoint [26]. Monte Carlo simulation provides great advantage using drug exposure-effect relationship [27] which considers pharmacokinetic variation in target animals, MIC distribution and PK/PD indices in defining the PK/PD cutoffs. The PK/PD cutoff of CEQ against $H$. parasuis was $0.06 \mathrm{mg} / \mathrm{L}$ under EMA recommended dosage $(2 \mathrm{mg} / \mathrm{kg} /$ day $)$. This $\mathrm{PK} / \mathrm{PD}$ cutoff value was similar with the clinical breakpoint values of cefotaxime, a third generation cephalosporin antibiotic, against Pasteurella multocida $(0.03 \mathrm{mg} / \mathrm{L})$ or Neisseria

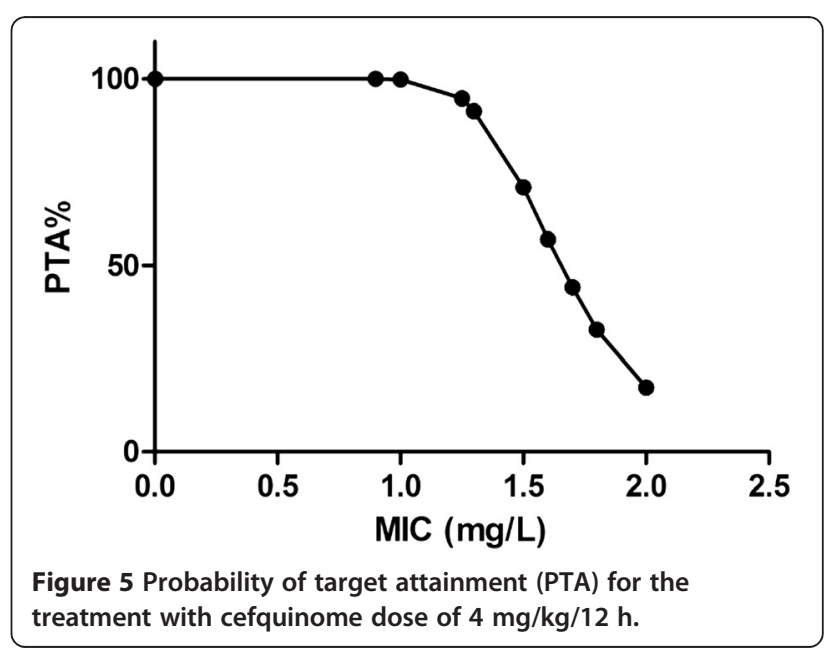


meningitides $(0.12 \mathrm{mg} / \mathrm{L})$. Similarities were also found in values of clinical breakpoints of cefotaxime $(0.12 \mathrm{mg} / \mathrm{L})$, ceftriaxone $(0.12 \mathrm{mg} / \mathrm{L})$, cefixime $(0.12 \mathrm{mg} / \mathrm{L})$, cefpodoxime $(0.25 \mathrm{mg} / \mathrm{L})$ and ceftaroline $(0.03 \mathrm{mg} / \mathrm{L})$ against Haemophilus influenzae, another bacteria of Haemophilus spp. in human clinic (EUCAST, 2014) [28]. However, this breakpoint was much lower than the PK/PD cutoff of cefotaxime $(1 \mathrm{mg} / \mathrm{L})$, ceftriaxone $(1 \mathrm{mg} / \mathrm{L})$, ceftazidime $(4 \mathrm{mg} / \mathrm{L})$ and cefepime $(4 \mathrm{mg} / \mathrm{L})$ (EUCAST, 2014) [28]. As the half-life and the PK/PD targets of CEQ are almost identical with those of the cephalosporins (1.5$2.5 \mathrm{~h})[25,29]$, the main reason for the difference may result from the dosing interval. PK/PD cutoff values for those drugs were based on drugs administered two or three times one day except ceftriaxone while single dose administration as recommended by EMA was chose in this study for CEQ. Compared to single dosing, the concentrations of these drugs after administration with multiple doses within $24 \mathrm{~h}$ could maintain a relatively high level, resulting in a higher MIC value of threshold for attaining the same T\% > MIC value. Though the PK/PD cutoff had been determined, to establish a susceptibility breakpoint of CEQ, the clinical data are needed.

Under recommended dose regimen of $2 \mathrm{mg} / \mathrm{kg} / 24 \mathrm{~h}$, the PTAs for reach the T\% $>$ MIC of 61 and 71 were $44.8 \%$ and $28.2 \%$. This seems to indicate that only less than half of the infections could be cured and less than a third of infections could be bacterial eradicated. In order to improve clinical response to therapy and reduce selection pressure for antimicrobial resistance during therapy, increasing doses were needed. With the increasing of the total amount of drug, the PTA increased accordingly in both single doses and 2 splitting dose regimens. However, the same daily amount of CEQ administered once-daily or twice-daily, such as $1 \mathrm{mg} / \mathrm{kg} / 12$ $\mathrm{h}$ and $2 \mathrm{mg} / \mathrm{kg} / 24 \mathrm{~h}$, resulted in different PTAs $(76.87 \%$ and $44.79 \%$, respectively) implying that splitting dose would reach higher PTA than a single dose administration. Moreover, compared to the dose splitting scenario, the PTA increased much less under single dosing, although the total amount of drug was increased. Therefore, dosing interval change, such as daily dose splitting, offers a much more attractive strategy in improving the clinical curing rate. Similar conclusion was conducted in cefprozil against $H$. influenzae that once daily dosing of cefprozil provided less activity compared with twice daily [30].

A theoretical regimen (i.e., $16 \mathrm{mg} / \mathrm{kg} / 12 \mathrm{~h}$ ) was recommended based on the PTA for T\%>MIC of 61 over $90 \%$. However, considering the economic impact and the therapeutic outcome, $4 \mathrm{mg} / \mathrm{kg} / 12 \mathrm{~h}$ is suggested as a more suitable dose regimen, which is less expensive for farmers, but its PTA (85.79\%) for T\% > MIC of 61 is closer to $90 \%$. Additionally, a much less drug amount dosage can be more eco-friendly, cost-effective and minimize the drug residue burden on public health via human food consumption.

Under the EMA recommended regimen, because of the single dose and short elimination half-life of CEQ, the derived PK/PD cutoff was $0.06 \mathrm{mg} / \mathrm{L}$. It was lower than $\mathrm{MIC}_{50}(0.125 \mathrm{mg} / \mathrm{L})$ of isolates from China. This result indicates that no more than half of the infection in China district could use CEQ for curing under recommend regimen. While, for the dose of $4 \mathrm{mg} / \mathrm{kg} / 12 \mathrm{~h}$ recommended in this study, the PK/PD cutoff was $1.3 \mathrm{mg} / \mathrm{L}$. Though it didn't cover all the MIC value, it covered more than $85 \%$ of the MIC distribution. Howerver, one point should be paid attention to is that the strains used in this simulation were limited in china. It cannot stand for the MIC distribution of the world. For international CEQ dose regimen against $H$. parasuis, more MICs of $H$. parasuis strains in a worldwide scale should be taken into account. Although further clinical studies are needed to confirm the modelling results, the present study could provide fundamental data for CEQ susceptibility test and improve the use of CEQ for swine health to a certain extent.

\section{Conclusions}

In conclusion, this study established an in vitro dynamic PK/PD modelling of CEQ against $H$. parasuis. The target values of CEQ for 3- $\log _{10}$-unit and 4- $\log _{10}$-unit decreases effects were T\% > MIC of 61 and 71 respectively. The very first CEQ PK/PD cutoff $(0.06 \mathrm{mg} / \mathrm{L})$ which is of great utility in CEQ susceptibility breakpoint determination and dosing design were derived based on Monte Carlo simulation. A more desirable dose regimen against $H$. parasuis was determined to be $4 \mathrm{mg} / \mathrm{kg} / 12 \mathrm{~h}$ in China.

\section{Abbreviations \\ T\% > MIC: The percent time that CEQ concentrations were above the minimum inhibitory concentration; H. parasuis: Haemophilus parasuis; CEQ: Cefquinome; PK/PD: Pharmacokinetic/pharmacodynamic; MIC: Minimal inhibitory concentrations; PRRSv: Porcine reproductive respiratory syndrome virus; EMA: The European Medicines Agency; BW: bodyweight; CLSI: The Clinical and Laboratory Standards Institute; VAST: Veterinary Antimicrobial Susceptibility Testing; EUCAST: The European Committee on Antimicrobial Susceptibility Testing; TSA: Tryptone soya agar; NAD: Beta-Nicotinamide adenine dinucleotide trihydrate; $\mathrm{MIC}_{50}$ and $\mathrm{MIC}_{90}$ : $\mathrm{MIC}$, inhibiting the growth of at least $50 \%$ and $90 \%$ of isolates in a test population; OD: Optical density; cfu: Colony forming unit; PTA: The probability of target attainment.}

\section{Competing interests}

The authors declare that they have no competing interests.

\section{Authors' contributions}

$Y L$ conceived of the study and participated in its design and coordination and helped to draft the manuscript. XX and JS design the experiment and draft the manuscript. XX, YC and $\mathrm{RH}$ carried out the MIC determination and in vitro time kill curve studies, GQ participated in the data analysis and revising the manuscript. HT and $\mathrm{YZ}$ carried out the bacteria isolation. All authors read and approved the final manuscript. 


\section{Acknowledgements}

This study was supported by the National Science Fund for Distinguished Young Scholars (Grant \# 31125026); Program for Changjiang Scholars and Innovative Research Team in University of Ministry of Education of China (Grant No. IRT13063). The funders had no role in study design, data collection and analysis, decision to publish, or preparation of the manuscript.

\section{Author details}

${ }^{1}$ College of Veterinary Medicine, National Reference Laboratory of Veterinary Drug Residues (SCAU), South China Agricultural University, Guangzhou 510642, China. ${ }^{2} 8725$, John J Kingman Rd, MS 6201, Ft Belvoir, VA 22060-6201, USA. ${ }^{3}$ Jiangsu Co-Innovation Centre for Prevention and Control of Important Animal Infectious Diseases and Zoonoses, Yangzhou, Jiangsu, People's Republic of China.

Received: 15 October 2014 Accepted: 30 January 2015

Published online: 13 February 2015

\section{References}

1. Oliveira S, Pijoan C. Haemophilus parasuis: new trends on diagnosis, epidemiology and control. Vet Microbiol. 2004;99(1):1-12.

2. Amano H, Shibata M, Kajio N, Morozumi T. Pathologic observations of pigs intranasally inoculated with serovar 1,4 and 5 of Haemophilus parasuis using immunoperoxidase method. J Vet Med Sci. 1994;56(4):639-44.

3. Frandoloso R, Pivato M, Martinez-Martinez S, Rodriguez-Ferri EF, Kreutz LC, Martin CB. Differences in Haemophilus parasuis adherence to and invasion of AOC-45 porcine aorta endothelial cells. BMC Vet Res. 2013;9:207.

4. Solano Gl, Segales J, Collins JE, Molitor TW, Pijoan C. Porcine reproductive and respiratory syndrome virus (PRRSv) interaction with Haemophilus parasuis. Vet Microbiol. 1997;55(1-4):247-57.

5. Zimmerman JJ, Yoon KJ, Wills RW, Swenson SL. General overview of PRRSV: a perspective from the United States. Vet Microbiol. 1997;55(1-4):187-96.

6. Kielstein P, Rapp-Gabrielson VJ. Designation of 15 serovars of Haemophilus parasuis on the basis of immunodiffusion using heat-stable antigen extracts. J Clin Microbiol. 1992;30(4):862-5.

7. Cai X, Chen H, Blackall PJ, Yin Z, Wang L, Liu Z, et al. Serological characterization of Haemophilus parasuis isolates from China. Vet Microbiol. 2005:111(3-4):231-6.

8. Angen O, Svensmark B, Mittal KR. Serological characterization of Danish Haemophilus parasuis isolates. Vet Microbiol. 2004;103(3-4):255-8.

9. Rapp-Gabrielson VJ, Gabrielson DA. Prevalence of Haemophilus parasuis serovars among isolates from swine. Am J Vet Res. 1992;53(5):659-64.

10. Morikoshi T, Kobayashi K, Kamino T, Owaki S, Hayashi S, Hirano S. Characterization of Haemophilus parasuis isolated in Japan. Nihon Juigaku Zasshi. 1990;52(3):667-9.

11. Rubies X, Kielstein P, Costa L, Riera P, Artigas C, Espuna E. Prevalence of Haemophilus parasuis serovars isolated in Spain from 1993 to 1997. Vet Microbiol. 1999;66(3):245-8.

12. Rafiee M, Blackall PJ. Establishment, validation and use of the KielsteinRapp-Gabrielson serotyping scheme for Haemophilus parasuis. Aust Vet J. 2000;78(3):172-4

13. Yu Y, Wu G, Zhai Z, Yao H, Lu C, Zhang W. Fifteen novel immunoreactive proteins of Chinese virulent Haemophilus parasuis serotype 5 verified by an immunoproteomic assay. Folia Microbiol. 2015;60(1):81-7.

14. The Europeam Medicines Agency. [http://www.ema.europa.eu/docs/en_GB/ document_library/Maximum_Residue_Limits_-_Report/2009/11/WC5000 11890.pdf]

15. Rey JF, Laffont CM, Croubels S, De Backer $\mathrm{P}$, Zemirline $\mathrm{C}$, Bousquet $\mathrm{E}$, et al. Use of Monte Carlo simulation to determine pharmacodynamic cutoffs of amoxicillin to establish a breakpoint for antimicrobial susceptibility testing in pigs. Am J Vet Res. 2014;75(2):124-31.

16. Zhang BX, Lu XX, Gu XY, Li XH, Gu MX, Zhang N, et al. Pharmacokinetics and ex vivo pharmacodynamics of cefquinome in porcine serum and tissue cage fluids. Vet J. 2014;199(3):399-405.

17. CLSI. Performance standards for antimicrobial disk and dilution susceptibility tests for bacteria isolated from animals. In: Approved Standard VET01-A4. 4th ed. Wayne, PA,USA: CLSI; 2013.

18. Schwarz S, Silley P, Simjee S, Woodford N, van Duijkeren E, Johnson AP, et al. Editorial: assessing the antimicrobial susceptibility of bacteria obtained from animals. J Antimicrob Chemother. 2010;65(4):601-4.

19. Zinner SH, Dudley M, Blaser J. In vitro models for the study of combination antibiotic therapy in neutropenic patients. Am J Med. 1986;80(6B):156-60.
20. Yang SS, Sun J, Liao XP, Liu BT, Li LL, Li L, et al. Co-location of the erm(T) gene and blaROB-1 gene on a small plasmid in Haemophilus parasuis of pig origin. J Antimicrob Chemother. 2013;68(8):1930-2.

21. Lees $P$, Svendsen $O$, Wiuff C. Strategies to minimize the impact of antimicrobial treatment on the selection of resistant bacteria. In: Guardabassi L, Jensen LB, Kruse H, editors. Guide to Antimicrobial Use in Animals (Chapter 6). Oxford: Wiley-Blackwell; 2008. p. 77-101.

22. MacGowan A, Bowker K. Developments in PK/PD: optimising efficacy and prevention of resistance. A critical review of PK/PD in in vitro models. Int J Antimicrob Agents. 2002;19(4):291-8.

23. Papich MG. Pharmacokinetic-pharmacodynamic (PK-PD) modeling and the rational selection of dosage regimes for the prudent use of antimicrobial drugs. Vet Microbiol. 2014;171(3-4):480-6.

24. Craig WA. Pharmacokinetic/pharmacodynamic parameters: rationale for antibacterial dosing of mice and men. Clin Infect Dis. 1998;26(1):1-10. quiz 11-12.

25. Craig WA. Interrelationship between pharmacokinetics and pharmacodynamics in determining dosage regimens for broad-spectrum cephalosporins. Diagn Microbiol Infect Dis. 1995;22(1-2):89-96.

26. Drusano GL, Preston SL, Hardalo C, Hare R, Banfield C, Andes D, et al. Use of preclinical data for selection of a phase II/III dose for evernimicin and identification of a preclinical MIC breakpoint. Antimicrob Agents Chemother. 2001;45(1):13-22.

27. Mueller M, de la Pena A, Derendorf H. Issues in pharmacokinetics and pharmacodynamics of anti-infective agents: kill curves versus MIC. Antimicrob Agents Chemother. 2004;48(2):369-77.

28. European Committee on Antimicrobial Susceptibility Testing. [http://www eucast.org/fileadmin/src/media/PDFs/EUCAST_files/Breakpoint_tables/ Breakpoint_table_v_4.0.pdf]

29. Balant L, Dayer P, Auckenthaler R. Clinical pharmacokinetics of the third generation cephalosporins. Clin Pharmacokinet. 1985;10(2):101-43.

30. Smith PF, Tsuji B, Booker BM, Forrest A, Bajic S, Kelchlin P, et al. Pharmacodynamics of cefprozil against Haemophilus influenzae in an in vitro pharmacodynamic model. Diagn Microbiol Infect Dis. 2006:56(4):379-86

\section{Submit your next manuscript to BioMed Central and take full advantage of:}

- Convenient online submission

- Thorough peer review

- No space constraints or color figure charges

- Immediate publication on acceptance

- Inclusion in PubMed, CAS, Scopus and Google Scholar

- Research which is freely available for redistribution 